OPEN ACCESS

Edited by:

Gianluca Rosso,

University of Turin, Italy

Reviewed by:

Alessandro Cuomo,

University of Siena, Italy

Virginio Salvi,

ASST Fatebenefratelli Sacco, Italy

${ }^{*}$ Correspondence: Myrthe G. B. M. Boekhorst

m.g.b.m.boekhorst@uvt.n

Specialty section:

This article was submitted to

Obstetric and Pediatric Pharmacology,

a section of the journal

Frontiers in Pediatrics

Received: 28 December 2020 Accepted: 25 February 2021

Published: 19 March 2021

Citation:

Boekhorst MGBM, Beerthuizen A, Hillegers M, Pop VJM and Bergink V (2021) Mother-to-Infant Bonding in Women With a Bipolar Spectrum Disorder. Front. Pediatr. 9:646985.

doi: 10.3389/fped.2021.646985

\section{Mother-to-Infant Bonding in Women With a Bipolar Spectrum Disorder}

\author{
Myrthe G. B. M. Boekhorst ${ }^{1,2 \star}$, Annemerle Beerthuizen ${ }^{1}$, Manon Hillegers ${ }^{3}$, \\ Victor J. M. Pop ${ }^{2}$ and Veerle Bergink ${ }^{1,4}$
}

'Department of Psychiatry, Erasmus MC, University Medical Center, Rotterdam, Netherlands, ${ }^{2}$ Department of Medical and Clinical Psychology, Tilburg University, Tilburg, Netherlands, ${ }^{3}$ Department of Child and Adolescent Psychiatry and Psychology, Erasmus MC, University Medical Center, Rotterdam, Netherlands, ${ }^{4}$ Departments of Psychiatry and Obstetrics, Icahn School of Medicine at Mount Sinai, New York, NY, United States

Purpose: Offspring of mothers with a bipolar disorder are at high-risk for impaired developmental outcomes and psychopathology (e. g., mood, anxiety, sleep disorders) later in life. This increased risk of psychopathology is not only because of genetic vulnerability, but environmental factors may play an important role as well. The often long and debilitating mood episodes of mothers with bipolar disorder might hamper their qualities as a caregiver and may impact the child. We examined early mother-to-infant bonding 1 year postpartum in mothers with bipolar spectrum disorder as compared to mothers of the general population. The association between mother-to-infant bonding and the type of bipolar spectrum diagnosis (bipolar I, bipolar II, bipolar Not Otherwise Specified) as well as relapse within 12 months postpartum was also assessed.

Methods: In total, 75 pregnant women with a bipolar spectrum disorder participated in the current study. The participants were included in a longitudinal cohort study of women with bipolar spectrum disorder and were prospectively followed from pregnancy until 1 year postpartum. Mother-to-infant bonding was assessed using the Pre- and Postnatal Bonding Scale. A longitudinal population-based cohort of 1,419 pregnant women served as the control group. Multiple linear regression analyses were used to assess the association between bipolar spectrum disorder and mother-to-infant bonding scores, controlling for several confounders.

Results: Women with bipolar spectrum disorder perceived the bonding with their child as less positive compared to the control group. The type of bipolar spectrum disorder was not associated with poorer bonding scores. Relapse during the 1st year after delivery also did not affect bonding scores in women with bipolar spectrum disorder.

Conclusions: Our findings could imply that women with bipolar spectrum disorder are more vulnerable to impairments in bonding due to the nature of their psychopathology, regardless of the occurrence of postpartum relapse. Careful follow-up including monitoring of mother-to-infant bonding of pregnant women with a history of bipolar spectrum disorder should be a standard to this vulnerable group of women. In addition, regardless of severity and mood episode relapse, an intervention to improve bonding could be beneficial for all mothers with bipolar spectrum disorder and their newborns.

Keywords: bonding, bipolar spectrum disorder, postpartum mania, perinatal, relapse 


\section{INTRODUCTION}

Children of mothers with bipolar spectrum disorder are a highrisk group to develop psychopathology later in life. Offspring of parents with bipolar disorder are not only at an increased risk of developing a mood disorder later in life $(1,2)$, but also other disorders such as anxiety disorders (2) and sleeping disorders $(3,4)$. Bipolar disorder has an impact on general child developmental outcomes of the offspring (e.g., motor quality (5), cognition (visual and verbal memory, processing speed, attention) (6), intellectual functioning after the onset of bipolar disorder (7) and psychosocial development [e.g., work, interpersonal relations, recreation, global functioning (8)].

Offspring of bipolar mothers are at increased risk of psychopathology because of their genetic vulnerability, but environmental factors are thought to play an important role as well $(3,9-13)$. The often long and debilitating mood episodes of mothers with bipolar disorder might hamper their qualities as a caregiver and can have an impact on the environment of the child. This can start early in life with impaired motherto-infant bonding (14). Mother-to-infant bonding is defined as "maternal feelings and emotions toward her infant" (15) (p1318). Impaired early mother-to-infant bonding has been found to be negatively associated with child behavioral and emotional development $(16,17)$, and higher levels of parenting stress during toddlerhood (18). In contrast to mother-toinfant bonding in women with bipolar disorder, mother-toinfant bonding in women with unipolar mood disorders has been well-studied. As expected, these studies have shown that depressive symptoms and depression, including postpartum depression, are associated with suboptimal early mother-toinfant bonding (19-24). Feeling inadequate, insecurity and negative cognitions are thought to influence bonding in mothers with depression (21).

Early mother-to-infant bonding in mothers with bipolar disorder has not been investigated, but a previous qualitative study described that mothers with bipolar disorder worried about potential problems in the relationship with their child. In this study, the majority of pregnant mothers with bipolar disorder were anxious to get a relapse postpartum potentially affecting them as a parent with some explicitly indicating concerns about bonding (25).

The aim of the current study was to examine motherto-infant bonding 1 year postpartum in a prospective cohort of women with a bipolar spectrum disorder who were followed since pregnancy. We compared motherto-infant bonding in mothers with a diagnosis in the bipolar spectrum [bipolar I (BD-I), bipolar II (BD-II), or bipolar Not Otherwise Specified (BD-NOS)] to mothers who participated in a large prospective population-based cohort, who were followed since pregnancy until 12 months postpartum in the Netherlands. As a next step, we aimed to investigate the association of mother-to-infant bonding with type of bipolar spectrum diagnosis and relapse within 12 months postpartum.

\section{MATERIALS AND METHODS}

\section{Participants and Procedure} Bipolar Spectrum Disorder Group

The participants of the current study were included in a longitudinal cohort study of women with $\mathrm{BD}$. Women were recruited via specialized bipolar disorder treatment centers. Inclusion criteria were a primary diagnosis of bipolar spectrum disorder (bipolar I (BD-I), bipolar II (BD-II), or bipolar Not Otherwise Specified (BD-NOS). DSM IV and DSM V classification systems do not have a distinct disease category for women with a history of a single episode of mania or psychosis after delivery, but no episodes at other times and we therefore classified these women as bipolar NOS. All women were diagnosed by a psychiatrist prior to their current pregnancy. Other inclusion criteria were age $\geq 18$, and a sufficient understanding of the Dutch language. Participants were approached by their psychiatrist and included between 2013 and 2018 , and were prospectively followed from the third trimester of pregnancy until 1 year postpartum. Enrolled pregnant women were treated as usual. All participants were asked to complete several (online) questionnaires (including a bonding scale) throughout the perinatal period. Only women who completed follow-up assessment at 12 months postpartum were selected for inclusion in the current sample. Of the 95 women who received this follow-up questionnaire, 20 (21\%) were lost to follow-up. This resulted in a total sample of 75 (79\%) women to be included for analyses in the current study. There were no differences in characteristics between the 75 included women, and the women who completed baseline assessment. The participating 75 women with bipolar spectrum disorder included 34 (45.3\%) women with a diagnosis of BD-I, 16 (21.3\%) women with a diagnosis $\mathrm{BD}-\mathrm{II}$ and, 25 (33.3\%) women with $\mathrm{BD}$ not otherwise specified, including 24 women with a history of postpartum mania, but no episodes at other times. Of these women, 63 (85.1\%) used psychotropic medication during their pregnancy. The study was approved by the Medical Ethics Committee at Erasmus University Medical Center, Rotterdam (MEC-2013319). All women provided written informed consent.

\section{Control Group}

Additionally, a population-based control group of 1,419 Dutchspeaking perinatal women were examined [HAPPY study; Truijens et al. (26)]. These women filled in the same questionnaires administered at equivalent time points. Details regarding the design of this study have been described elsewhere (26). In short, exclusion criteria were a diagnosis of a severe psychiatric disorder (e.g., schizophrenia, borderline personality disorder and bipolar disorder), use of psychotropic medicine during pregnancy (e.g., antidepressants, SSRIs, benzodiazepines), twin or multiple pregnancies and a history of chronic disease (e.g., diabetes and thyroid dysfunction). This longitudinal cohort study followed women from the first trimester of pregnancy until 1 year postpartum. Throughout this period, women were asked to complete (online) questionnaires regarding their psychological and physical well-being. The study was approved 
by the Psychology Ethics Committee at Tilburg University (protocol number EC-2012.25), and reviewed by the Medical Ethics Committee at the Máxima Medical Center, Veldhoven. All women provided written informed consent.

\section{Measures}

\section{Mother-to-Infant Bonding}

At 12 months postpartum, all mothers completed the Pre- and Postnatal Bonding Scale $(19,27)$, a self-report measure to assess feelings of bonding from a mother toward her infant. The scale consists of positive items assessing the core concept of love as a domain of bonding, and reflects positive feelings a mother might feel toward her child. Mothers were asked to rate items on a four-point Likert-type scale $(0=$ "not at all" to $3=$ "very much'). Higher scores indicate more positive feelings of bonding. The PPBS showed adequate psychometric properties, with a Cronbach's alpha of $0.80(19,27)$.

\section{Descriptive Characteristics Recent Depressive Symptomology}

At 12 months postpartum, mothers completed a screening question on their recent depressive symptomatology. Mothers indicated whether they had felt sad, miserable, or very anxious in the postpartum period for a period of at least 2 weeks, providing additional information on psychological well-being. Experience of recent depressive symptomatology was recoded into a dichotomous variable (yes/no).

\section{Depressive Symptoms}

In order to evaluate and compare the occurrence of depressive symptoms in both groups during late pregnancy (32 weeks) and early postpartum (6 weeks) we assessed depressive symptoms using the Dutch version of the Edinburgh Postnatal Depression Scale (EPDS) (28). The EPDS is a self-report measure and has been validated for use in non-postnatal women (29), as well as for use in a Dutch population, both during pregnancy (30) and postpartum (31). Items are rated on four-point Likert-type scales, with total scores ranging from 0 to 30. Higher scores indicate a greater level of depressive symptoms. At 12 months postpartum, the control group completed the EPDS, while women with a bipolar spectrum disorder completed this assessment when they indicated that they experienced recent depressive symptomatology. Furthermore, at 12 months postpartum, women were asked if they had experienced a relapse the 1st year postpartum and the severity of this episode was assessed using the EPDS (32).

\section{Demographic Variables}

Data regarding demographics and obstetric information were collected with online questionnaires in both mothers with a bipolar spectrum disorder and controls. In particular: age (in years), educational level (high: a Bachelor or Master's degree), having a partner (yes or no), and employment (yes or no). Furthermore, obstetric information was collected, such as parity (primiparous or multiparous), pregnancy intention (planned or unplanned), previous miscarriage or abortion (yes or no), and the gender of the child.

In the cohort of women with a bipolar spectrum disorder, information concerning the diagnosis and additional details with regard to a postpartum relapse (symptoms of depression, mania, psychosis) were obtained from their medical records.

\section{Statistical Analyses}

Variables were compared between the control group and the bipolar spectrum disorder group using Chi-square tests for categorical variables and $t$-tests for continuous variables. If statistically significant, the strength of the relationship was expressed as Cramer's coefficients or Cohen's $d$ and considered small or medium following conventional criteria.

First, we used multiple linear regression to assess the association between the maternal group (independent variable) and mother-to-infant bonding scores (dependent variable), adjusting for potential predetermined covariates based on previous studies (recent depressive symptomology, age, level of education, parity and unplanned pregnancy) (19).

Next, the group of women with a bipolar spectrum disorder was further analyzed to assess the association with motherto-infant bonding within specific subgroups, such as primary diagnosis (bipolar disorder I (BD-I), bipolar disorder II (BD-II), or history of postpartum mania and perinatal relapse (yes/no). Relapse rate was assessed and compared between subgroups of primary diagnosis using Chi-square tests. Depressive symptoms during pregnancy and early postpartum were also compared between women with and without a relapse, using $t$-test. Multiple linear regression analyses were also conducted to assess these associations. For multiple linear regression analyses, the BD-I, BD-II, and postpartum mania group were coded into dichotomous variables, while the variable age was centered on the mean. Statistical analyses were performed using IBM SPSS version 26.0.

\section{RESULTS}

The demographic and obstetric characteristics of the bipolar spectrum disorder group and the control group are shown in Table 1. Differences were found with regard to age and parity between the two groups, with women with bipolar spectrum disorder being slightly older (medium effect size) and more often multiparous. The control group had a paid job more often. Compared to the control group, more women with bipolar spectrum disorder reported that their pregnancy was unplanned. Apart from age, all differences had a small effect size. The groups did not differ with regard to level of education, previous miscarriage or abortion, having a partner, and prenatal and postnatal depressive symptoms.

Furthermore, of the mothers with a bipolar spectrum disorder, 29 (38.7\%) experienced recent depressive symptomatology, while only $35(2.5 \%)$ of the control group experienced these symptoms $\left[X_{(1,1,494)}^{2}=227.67, p<0.00, \mathrm{Phi}=-0.390\right.$, medium effect size $]$. 
TABLE 1 | Demographic, psychiatric and obstetric characteristics of the participating women.

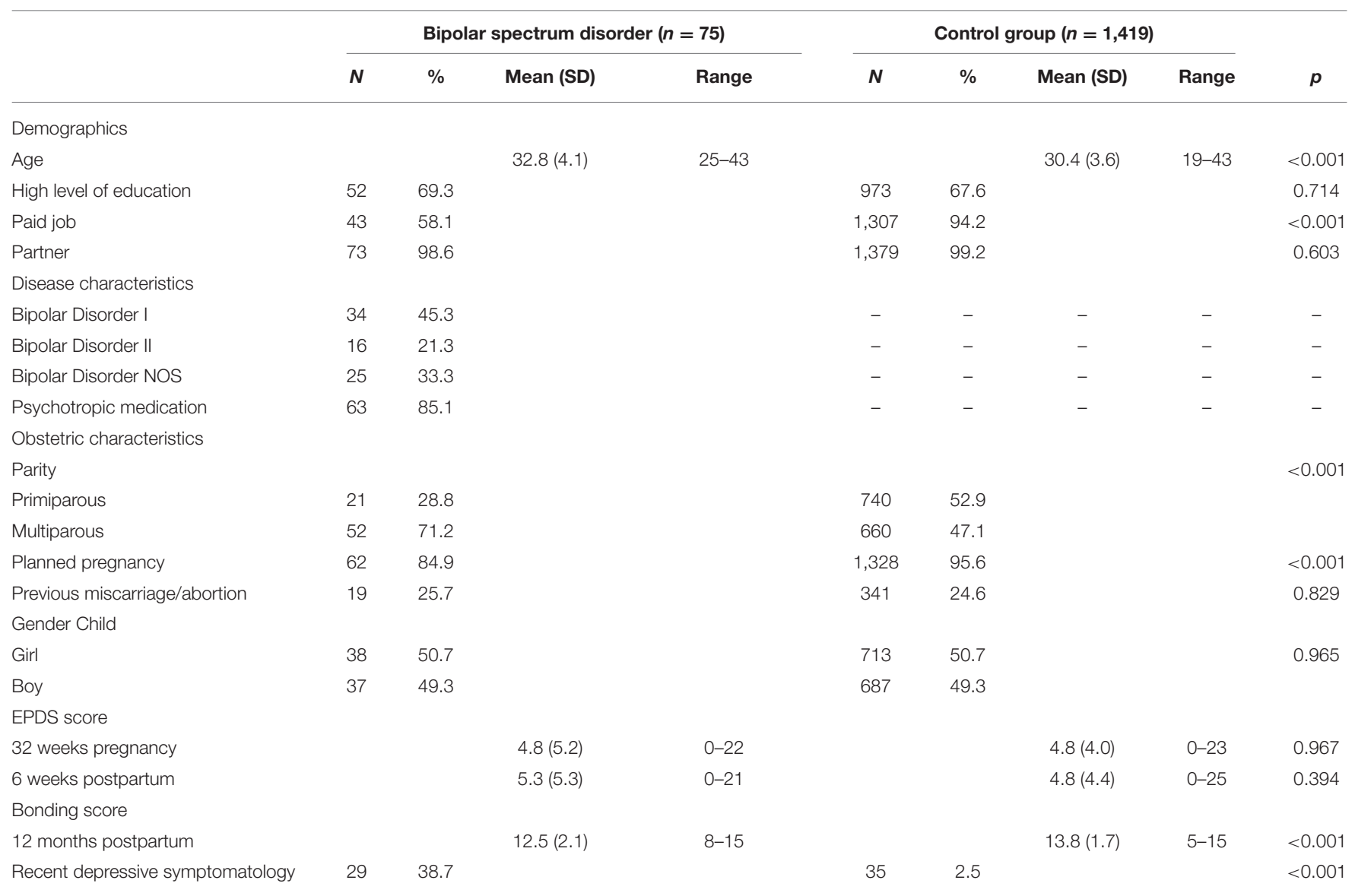

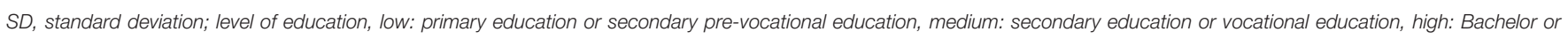
Master's degree; EPDS, Edinburgh Postnatal Depression Scale; higher bonding scores indicate more positive feelings of bonding.

TABLE 2 | Multiple regression predicting mother-to-infant bonding $(N=1,445)$.

\begin{tabular}{|c|c|c|c|c|}
\hline & B (SE) & $\beta$ & $t$ & $p$ \\
\hline Bipolar spectrum disorder & $-0.89(0.23)$ & -0.11 & -3.95 & $<0.001$ \\
\hline Recent depressive symptomatology & $-0.76(0.24)$ & -0.09 & -3.18 & 0.002 \\
\hline Maternal age & $-0.002(0.01)$ & -0.01 & -0.17 & 0.869 \\
\hline High level of education & $-0.49(0.10)$ & -0.13 & -4.85 & $<0.001$ \\
\hline Multiparous & $-0.33(0.09)$ & -0.10 & -3.57 & $<0.001$ \\
\hline
\end{tabular}

$B$, unstandardized regression coefficient; SE, standard error; $\beta$, standardized regression coefficient.

\section{Group Differences in Mother-to-Infant Bonding BD Group vs. Control Group}

Mean bonding scores differed between groups $(t=5.17$, $p<0.001$, Cohen's $d=0.67$, medium effect size), with women in the bipolar spectrum disorder group reporting lower mother-toinfant bonding scores $(\mathrm{M}=12.46, \mathrm{SD}=2.13)$ compared to the control group $(\mathrm{M}=13.76, \mathrm{SD}=1.68)$. Results of the multiple linear regression model predicting mother-to-infant bonding showed that the total model was significant, $\mathrm{F}_{(6,1,445)}=15.86$, $p<0.001, R^{2}=0.062$. Bipolar spectrum disorder was independently associated with poorer mother-to-infant bonding scores compared to the control group after adjustment for recent depressive symptomatology, age, education, parity and unplanned pregnancy $(\beta=-0.11, \mathrm{SE}=0.23, p<0.001)$, see Table 2.

\section{Group Differences Among Mothers With Different Types of Bipolar Spectrum Disorder}

Mothers with a BD-I or BD-II diagnosis reported higher mean bonding scores (more positive bonding) 12 months postpartum 
$(\mathrm{M}=12.76, \mathrm{SD}=2.09$, and $\mathrm{M}=12.88, \mathrm{SD}=1.96$, respectively) compared to women with a history of postpartum mania $(\mathrm{M}=11.67, \mathrm{SD}=2.14)$. $(t=1.95, p=0.056$, Cohen's $\mathrm{d}=0.52$, medium effect size and, $t=1.81, p=0.079$, Cohen's $\mathrm{d}=0.59$, medium effect size, respectively). There was no difference in mean bonding scores between the BDI and BD-II group $(t=0.18, p=0.86)$. After adjustment for recent depressive symptomatology, age, education, parity and unplanned pregnancy, the primary diagnosis was not independently associated with poorer bonding scores (BD-I: $\beta$ $=0.21, \mathrm{SE}=0.68, p=0.191$ and $\mathrm{BD}-\mathrm{II}: \beta=0.17, \mathrm{SE}=0.85$, $p=0.306)$.

\section{Effect of Postpartum Relapse on Mother-to-Infant Bonding}

In total, $27(37.5 \%)$ women had a relapse within 12 months after giving birth, defined as a depressive episode, hypo(-mania) and/or psychosis. There was a significant difference in mean EPDS scores at 6 weeks postpartum between women with a relapse $(\mathrm{M}=7.72, \mathrm{SD}=5.83)$ and women without a relapse $(\mathrm{M}=4.10, \mathrm{SD}=4.58)(t=2.79, p=0.007$, Cohen's $\mathrm{d}=0.72$, medium effect size). With regard to the primary diagnosis, 10 (31.3\%) of the women with BD-I, 9 (56.3\%) of the women with BD-II and, 8 (34.8\%) of the women with a history of postpartum mania relapsed during the current postpartum period.

Mean bonding scores did not differ between women with ( $M$ $=12.22, \mathrm{SD}=2.21)$ and without a relapse $(\mathrm{M}=12.64, \mathrm{SD}=$ 2.08) $(t=0.82, p=0.418)$.

\section{DISCUSSION}

The current study aimed to assess mother-to-infant bonding in mothers with a bipolar spectrum disorder. The present study showed that women with bipolar spectrum disorder showed worse bonding scores at 12 months postpartum compared to postpartum population-based control women, after controlling for several potential confounders. The type of the primary diagnosis (bipolar I disorder, bipolar II disorder or bipolar NOS, including women with a history of postpartum mania) was not associated with impaired bonding. The occurrence of relapse in the perinatal period did not influence the bonding score, which was unexpected.

Our findings add to the knowledge that women with bipolar spectrum disorder and their offspring are a vulnerable and highrisk group, providing a first indication of a suboptimal motherto-infant bonding early in life. Possibly, women with bipolar spectrum disorder have increased fears of failure with regard to bonding, as well as feelings of insecurity, which may impact their perceptions of the bond with their child. These worries have been highlighted in the results of a recent qualitative study, showing that mothers with a bipolar disorder feared their mood episodes would affect bonding with their child (e.g., not wanting their child) (25).

The postpartum relapse rate in our study was high $(37.5 \%)$ but in line with our prior meta-analysis in which we reported an overall postpartum relapse risk of $35 \%(95 \% \mathrm{CI}=29$,
41) (33). Interestingly, relapse during the current postpartum period did not affect bonding scores in women with a bipolar spectrum disorder. Our findings could imply that women with a bipolar spectrum disorder are more vulnerable to impairments in bonding due to the nature of their disorder, regardless of relapse. Similar to mothers with depression, feelings of inadequacy, insecurity and negative cognitions (21) could influence bonding in mothers with a bipolar spectrum disorder. Due to the small sample of women with relapse, we were unable to assess differences in bonding regarding the severity, timing, and symptomatology of relapse (depressive symptoms, mixed episode or psychosis/mania). Studies that have compared mother-toinfant bonding in patients with postpartum depression and postpartum psychosis who had been admitted to a mother-baby unit, found that especially women with postpartum depression perceive impairments in bonding $(21,22)$. Notably, these studies did not include a population of patients diagnosed with a bipolar spectrum disorder, nor a control group, making it difficult to compare them with the findings of the current study.

The current study has several strengths and limitations. The strength of the study is the case-control design of participants who were longitudinally studied during pregnancy and in the postpartum period. Our study also has limitations that should be considered when interpreting the results. First, the bonding questionnaire is a short screening instrument examining the core concept of mother-to-infant bonding (core concept of love), and shows good psychometric properties and reliability $(19,27)$. Future research is needed to more extensively assess differences in various components of early bonding (e.g., rejection, anxiety about care) in mothers with a bipolar spectrum disorder. A major limitation of the study is the lack of assessment on current mood status. Depressive symptoms were assessed using the EPDS at 12 months postpartum, in those women with a relapse only. Although current symptomatology was not measured using a standard assessment (e.g., EPDS), recent depressive symptomatology during the postpartum period was assessed using a screening question, which was included in the analyses as a covariate. Mothers with a bipolar spectrum disorder reported poorer mother-to-infant bonding scores, even after controlling for depressive symptomatology. Furthermore, the bonding scores did not differ between mothers with a bipolar spectrum disorder who experienced recent depressive symptomatology compared to mothers without these symptoms. For the screening question, women reflected on their depressive symptomatology over the entire postpartum period. Even so, we expect that a telescoping effect may have occurred (34), causing women to have reflected on more recent symptoms when completing this screening question, rather than symptoms occurring more in the past (e.g., early postpartum). This may have led to participants reporting more current symptomatology. Nonetheless, we recommend that this effect is further investigated and validated in other studies and groups. Another limitation of the current study is that both women in the control cohort and the bipolar spectrum disorder cohort were more often highly educated compared to the general Dutch population (35) and were predominantly Caucasian which hampers generalizability of our results. Moreover, $21 \%$ of women with a bipolar spectrum disorder were lost to follow-up, and 
therefore did not complete bonding assessment at 12 months postpartum, leading to incomplete follow-up of these women in the study over time. Lastly, the diagnosis of an episode of relapse was made by the treating psychiatrist and not by a formal diagnostic interview. The study was designed to limit the burden of study participation in a vulnerable group of pregnant and postpartum women with the aim to recruit a representative sample of bipolar women in terms of disease severity, and not only women with relatively mild or stable bipolar disorder who are willing to do extra in person diagnostic interviews for research purposes.

The current findings suggest that women with a history of bipolar spectrum disorder prior to the current pregnancy, regardless of relapse during the current postpartum period, are at increased risk for impaired mother-to-infant bonding. Our findings suggest that incorporating aspects of bonding in interventions for mothers with a history of a bipolar spectrum disorder could be valuable, irrespective of a relapse. For example, Practical Resources for Effective Postpartum Parenting is an intervention for women at risk for postpartum depression that focuses on the mother-infant dyad, and had positive outcomes for the mother, child and the mother-child relationship (36). Next, findings of a meta-analysis showed that maternal-child interaction guidance could be a promising intervention for mothers with postpartum depression, with regard to parenting and child outcomes (37). Future studies should address the effectiveness of such interventions for bipolar disorder motherinfant dyads. Suboptimal bonding is a risk factor for negative behavioral and emotional outcomes in children $(16,17)$. It is therefore important to integrate interventions to improve bonding in the treatment of mothers with a bipolar spectrum disorder. It is also of importance that polyclinic and obstetric health care facilities provide follow-up for parents with mental illness and their newborn. Especially because offspring of parents with bipolar disorder are at ultra-high risk for developing a mood disorder and other disorders later in life (1-3). Mother-to-infant bonding is a process that can already begin prenatally (38) and

\section{REFERENCES}

1. Mesman E, Nolen WA, Reichart CG, Wals M, Hillegers MHJ. The dutch bipolar offspring study: 12-year follow-up. Am J Psychiatry. (2013) 170:5429. doi: 10.1176/appi.ajp.2012.12030401

2. Lau P, Hawes DJ, Hunt C, Frankland A, Roberts G, Mitchell PB. Prevalence of psychopathology in bipolar high-risk offspring and siblings: a meta-analysis. Eur Child Adolesc Psychiatry. (2018) 27:823-37. doi: 10.1007/s00787-017-1050-7

3. Sandstrom A, Sahiti Q, Pavlova B, Uher R. Offspring of parents with schizophrenia, bipolar disorder, and depression: a review of familial high-risk and molecular genetics studies. Psychiatr Genet. (2019) 29:1609. doi: 10.1097/YPG.0000000000000240

4. Wescott DL, Morash-Conway J, Zwicker A, Cumby J, Uher R, Rusak B. Sleep in offspring of parents with mood disorders. Front Psychiatry. (2019) 10:1-8. doi: 10.3389/fpsyt.2019.0 0225

5. Santucci AK, Singer LT, Wisniewski SR, Luther JF, Eng HF, Sit DK, et al. Oneyear developmental outcomes for infants of mothers with bipolar disorder. $J$ Clin Psychiatry. (2017) 78:1083-90. doi: 10.4088/JCP.15m10535 future research could focus on the best timing and type of intervention to improve bonding.

\section{DATA AVAILABILITY STATEMENT}

The data can be requested from the authors. Requests to access the datasets should be directed to m.g.b.m.boekhorst@uvt.nl.

\section{ETHICS STATEMENT}

The studies involving human participants were reviewed and approved by Medical Ethics Committee at Erasmus University Medical Center, Rotterdam MEC-2013-319 Psychology and Ethics Committee at Tilburg University EC-2012.25. The patients/participants provided their written informed consent to participate in this study.

\section{AUTHOR CONTRIBUTIONS}

$\mathrm{MB}$ contributed to the hypotheses, data analyses and wrote the paper. $\mathrm{AB}$ contributed to the hypotheses and collaborated in writing and editing of the paper. $\mathrm{MH}$ and $\mathrm{VP}$ contributed to the hypotheses and collaborated in writing and editing of the paper. VB is the principal investigator and contributed to funding acquisition, material preparation, process of data collection, hypotheses, data analyses and collaborated in writing and editing of the paper. All authors read and approved the final version of the manuscript.

\section{FUNDING}

This work was supported by Department of Psychiatry, Erasmus MC, University Medical Center Rotterdam, the Netherlands, and by Department of Child and Adolescent Psychiatry and Psychology, Erasmus MC, University Medical Center Rotterdam, the Netherlands.
6. Bora E, Özerdem A. A meta-analysis of neurocognition in youth with familial high risk for bipolar disorder. Eur Psychiatry. (2017) 44:1723. doi: 10.1016/j.eurpsy.2017.02.483

7. Klimes-Dougan B, Jeong J, Kennedy KP, Allen TA. Intellectual functioning in offspring of parents with bipolar disorder: a review of the literature. Brain Sci. (2017) 7:21-3. doi: 10.3390/brainsci7110143

8. Bella T, Goldstein T, Axelson D, Obreja M, Monk K, Hickey MB, et al. Psychosocial functioning in offspring of parents with bipolar disorder. J Affect Disord. (2011) 133:204-11. doi: 10.1016/j.jad.2011.03.022

9. Iacono V, Beaulieu L, Hodgins S, Ellenbogen MA. Parenting practices in middle childhood mediate the relation between growing up with a parent having bipolar disorder and offspring psychopathology from childhood into early adulthood. Dev Psychopathol. (2018) 30:635-49. doi: 10.1017/S095457941700116X

10. Freed RD, Tompson MC, Otto MW, Nierenberg AA, Hirshfeld-Becker D, Wang $\mathrm{CH}$, et al. Early risk factors for psychopathology in offspring of parents with bipolar disorder: the role of obstetric complications and maternal comorbid anxiety. Depress Anxiety. (2014) 31:583-90. doi: 10.1002/da.22254

11. Schreuder MM, Vinkers $\mathrm{CH}$, Mesman E, Claes S, Nolen WA, Hillegers MHJ. Childhood trauma and HPA axis functionality 
in offspring of bipolar parents. Psychoneuroendocrinology. 74:316-23. doi: 10.1016/j.psyneuen.2016.09.017

12. Koenders MA, Mesman E, Giltay EJ, Elzinga BM, Hillegers MHJ. Traumatic experiences, family functioning, and mood disorder development in bipolar offspring. Br J Clin Psychol. (2020) 59:277-89. doi: 10.1111/bjc.12246

13. Doucette S, Levy A, Flowerdew G, Horrocks J, Grof P, Ellenbogen M, et al. Early parent-child relationships and risk of mood disorder in a Canadian sample of offspring of a parent with bipolar disorder: findings from a 16-year prospective cohort study. Early Interv Psychiatry. (2016) 10:3819. doi: 10.1111/eip.12195

14. Winston $\mathrm{R}$, Chicot $\mathrm{R}$. The importance of early bonding on the long-term mental health and resilience of children. London J Prim Care (Abingdon). (2016) 8:12-4. doi: 10.1080/17571472.2015.1133012

15. Bicking Kinsey C, Hupcey JE. State of the science of maternal-infant bonding: a principle-based concept analysis. Midwifery. (2013) 29:131420. doi: 10.1016/j.midw.2012.12.019

16. Fuchs A, Möhler E, Reck C, Resch F, Kaess M. The early mother-tochild bond and its unique prospective contribution to child behavior evaluated by mothers and teachers. Psychopathology. (2016) 49:2116. doi: 10.1159/000445439

17. Mason ZS, Briggs RD, Silver EJ. Maternal attachment feelings mediate between maternal reports of depression, infant social-emotional development, and parenting stress. J Reprod Infant Psychol. (2011) 29:382-94. doi: 10.1080/02646838.2011.629994

18. de Cock ESA, Henrichs J, Klimstra TA, Janneke A, Vreeswijk CMJM, Meeus WHJ, et al. Longitudinal associations between parental bonding, parenting stress, and executive functioning in toddlerhood. J Child Fam Stud. (2017) 26:1723-33. doi: 10.1007/s10826-017-0679-7

19. Cuijlits I, de Wetering AP, Endendijk JJ, Baar AL, Potharst ES, Pop VJM. Risk and protective factors for pre- and postnatal bonding. Infant Ment Health J. (2019) 40:768-85. doi: 10.1002/imhj.21811

20. Dubber S, Reck C, Müller M, Gawlik S. Postpartum bonding: the role of perinatal depression, anxiety and maternal-fetal bonding during pregnancy. Arch Womens Ment Health. (2015) 18:187-95. doi: 10.1007/s00737-014-0445-4

21. Hornstein C, Trautmann-Villalba P, Hohm E, Rave E, Wortmann-Fleischer S, Schwarz M. Maternal bond and mother-child interaction in severe postpartum psychiatric disorders: Is there a link? Arch Womens Ment Health. (2006) 9:279-84. doi: 10.1007/s00737-006-0148-6

22. Noorlander Y, Bergink V, Van Den Berg MP. Perceived and observed mother-child interaction at time of hospitalization and release in postpartum depression and psychosis. Arch Womens Ment Health. (2008) 11:4956. doi: 10.1007/s00737-008-0217-0

23. Tichelman E, Westerneng $M$, Witteveen $A B$, van Baar $A L$, van der Horst HE, de Jonge A, et al. Correlates of prenatal and postnatal mother-to-infant bonding quality: a systematic review. PLoS ONE. (2019) 14:e0222998. doi: 10.1371/journal.pone.0222998

24. O'Higgins M, Roberts ISJ, Glover V, Taylor A. Mother-child bonding at 1 year; Associations with symptoms of postnatal depression and bonding in the first few weeks. Arch Womens Ment Health. (2013) 16:381-9. doi: 10.1007/s00737-013-0 354-y

25. Anke TMS, Slinning K, Skjelstad DV. "What if I get ill?" perinatal concerns and preparations in primi- and multiparous women with bipolar disorder. Int J Bipolar Disord. (2019) 7:7. doi: 10.1186/s40345-019-0 143-2
26. Truijens SEM, Meems M, Kuppens SMI, Broeren MAC, Nabbe KCAM, Wijnen HA, et al. The HAPPY study (Holistic Approach to Pregnancy and the first Postpartum Year): design of a large prospective cohort study. BMC Pregnancy Childbirth. (2014) 14:1-12. doi: 10.1186/1471-2393-14-312

27. Cuijlits I, Van de Wetering A, Potharst E, Truijens S, Van Baar A, Pop V. Development of a Pre- and Postnatal Bonding Scale (PPBS). J Psychol Psychother. (2016) 6:282. doi: 10.4172/2161-0487.1000282

28. Cox JL, Holden JM, Sagovsky R. Detection of postnatal depression: development of the 10-item Edinburgh Postnatal Depression Scale. $\mathrm{Br} J$ Psychiatry. (1987) 150:782-6. doi: 10.1192/bjp.150.6.782

29. Cox JL, Chapman G, Murray D, Jones P. Validation of the Edinburgh Postnatal Depression Scale (EPDS) in non- postnatal women. J Affect Disord. (1996) 39:185-9. doi: 10.1016/0165-0327(96)00008-0

30. Bergink V, Kooistra L, Lambregtse-van den Berg MP, Wijnen H, Bunevicius R, van Baar A, et al. Validation of the Edinburgh Depression Scale during pregnancy. J Psychosom Res. (2011) 70:385-9. doi: 10.1016/j.jpsychores.2010.07.008

31. Pop VJ, Komproe IH, van Son MJ. Characteristics of the Edinburgh PostNatal Depression Scale in The Netherlands. J Affect Disord. (1992) 26:10510. doi: 10.1016/0165-0327(92)90041-4

32. Meltzer-Brody S, Boschloo L, Jones I, Sullivan PF, Penninx BW. The EPDSlifetime: assessment of lifetime prevalence and risk factors for perinatal depression in a large cohort of depressed women. Arch Womens Ment Health. (2013) 16:465-73. doi: 10.1007/s00737-013-0372-9

33. Wesseloo R, Kamperman AM, Munk-Olsen T, Pop VJM, Kushner SA, Bergink $\mathrm{V}$. Risk of postpartum relapse in bipolar disorder and postpartum psychosis: a systematic review and meta-analysis. Am J Psychiatry. (2016) 173:11727. doi: 10.1176/appi.ajp.2015.15010124

34. Barsky AJ. Forgetting, fabricating, and telescoping. Arch Intern Med. (2002) 162:981. doi: 10.1001/archinte.162.9.981

35. Statistics the Netherlands. Population; Educational Level; Gender, Age and Migration Background (Bevolking; Onderwijsniveau; Geslacht, Leeftijd en Migratieachtergrond). (2019). Available online at: https://opendata.cbs.nl/ statline/\#/CBS/nl/dataset/82275NED/table?fromstatweb (accessed October 22, 2019).

36. Werner EA, Gustafsson HC, Lee S, Feng T, Jiang N, Desai P, et al. PREPP: postpartum depression prevention through the mother-infant dyad. Arch Womens Ment Health. (2016) 19:229-42. doi: 10.1007/s00737-015-0549-5

37. Letourneau NL, Dennis CL, Cosic N, Linder J. The effect of perinatal depression treatment for mothers on parenting and child development: a systematic review. Depress Anxiety. (2017) 34:928-66. doi: 10.1002/da.22687

38. de Cock ESA, Henrichs J, Vreeswijk CMJM, Maas AJBM, Rijk CHAM, van Bakel HJA. Continuous feelings of love? The parental bond from pregnancy to toddlerhood. J Fam Psychol. (2016) 30:125-34. doi: 10.1037/fam0000138

Conflict of Interest: The authors declare that the research was conducted in the absence of any commercial or financial relationships that could be construed as a potential conflict of interest.

Copyright (C) 2021 Boekhorst, Beerthuizen, Hillegers, Pop and Bergink. This is an open-access article distributed under the terms of the Creative Commons Attribution License (CC BY). The use, distribution or reproduction in other forums is permitted, provided the original author(s) and the copyright owner(s) are credited and that the original publication in this journal is cited, in accordance with accepted academic practice. No use, distribution or reproduction is permitted which does not comply with these terms. 